\title{
A novel combinatorial technique for simultaneous quantification of oxygen radicals and aggregation reveals unexpected redox patterns in the activation of platelets by different physiopathological stimuli
}

Dina Vara ${ }^{1}$ Eugenia Cifuentes-Pagano ${ }^{2}$ Patrick J. Pagano ${ }^{2}$ and Giordano Pula

${ }^{1}$ Institute of Biomedical and Clinical Science, University of Exeter Medical School, Exeter, UK and ${ }^{2}$ Department of Pharmacology and Chemical Biology and Vascular Medicine Institute, School of Medicine, University of Pittsburgh, Pittsburgh, PA, USA

\section{ABSTRACT}

T The regulation of platelets by oxidants is critical for vascular health and may explain thrombotic complications in diseases such as diabetes and dementia, but remains poorly understood. Here, we describe a novel technique combining electron paramagnetic resonance spectroscopy and turbidimetry, which has been utilized to monitor simultaneously platelet activation and oxygen radical generation. This technique has been used to investigate the redox-dependence of human and mouse platelets. Using selective peptide inhibitors of NADPH oxidases (NOXs) on human platelets and genetically modified mouse platelets $\left(\mathrm{NOX}^{-/}\right.$or $\left.\mathrm{NOX}^{-/}\right)$, we discovered that: 1) intracellular but not extracellular superoxide anion generated by NOX is critical for platelet activation by collagen; 2) superoxide dismutation to hydrogen peroxide is required for thrombin-dependent activation; 3) NOX1 is the main source of oxygen radicals in response to collagen, while NOX2 is critical for activation by thrombin; 4) two platelet modulators, namely oxidized low density lipoproteins (oxLDL) and amyloid peptide $\beta(A \beta)$, require activation of both NOX1 and NOX2 to pre-activate platelets. This study provides new insights into the redox dependence of platelet activation. It suggests the possibility of selectively inhibiting platelet agonists by targeting either NOX1 (for collagen) or NOX2 (for thrombin). Selective inhibition of either NOX1 or NOX2 impairs the potentiatory effect of tested platelet modulators (oxLDL and A $\beta$ ), but does not completely abolish platelet hemostatic function. This information offers new opportunities for the development of disease-specific antiplatelet drugs with limited bleeding side effects by selectively targeting one NOX isoenzyme.

\section{Introduction}

Platelets are anucleated circulating cells responsible for initiating hemostasis via thrombus formation and blood clotting. The regulation of platelets is of primary importance for cardiovascular medicine and for the discovery of new drugs to treat cardiovascular diseases. ${ }^{1}$ In addition to canonical signaling pathways depending on protein kinase activity, ${ }^{2}$ platelets are regulated in a redox-dependent manner. Several lines of evidence suggest that platelets are modulated by extracellular reactive oxygen species $(\mathrm{ROS})^{3}$ and that platelet activation is essentially dependent on the generation of endogenous ROS. ${ }^{4-6}$ Therefore, the study of platelet regulation and hemostasis is shedding light on the interface between ROS biochemistry and cellular physiology. Superoxide anion $\left(\mathrm{O}_{2}{ }^{-}\right)$from exogenous sources or endogenously produced by platelets is shown to significantly increase platelet aggregation and thrombus formation. $\mathrm{O}_{2}{ }^{--}$has a pre-eminent role in biology and pathophysiology, as it serves as a progenitor for formation of hydrogen peroxide $\left(\mathrm{H}_{2} \mathrm{O}_{2}\right)$, peroxynitrite $\left(\mathrm{ONO} \mathrm{O}^{--}\right)$and hydroxyl radical $\left(\mathrm{HO}^{\circ}\right)$, and thereby plays a key role in the post-translational oxidative modification of proteins. ${ }^{8}$ The work of several research groups has focused on
Ferrata Storti Foundation

Haematologica 2019

Volume 104(9):1879-1891

\section{Correspondence:}

GIORDANO PULA

g.pula@exeter.ac.uk

Received: October 8, 2018.

Accepted: January 18, 2019.

Pre-published: January 24, 2019.

doi:10.3324/haematol.2018.208819

Check the online version for the most updated information on this article, online supplements, and information on authorship \& disclosures: www.haematologica.org/content/104/9/1879

(C)2019 Ferrata Storti Foundation

Material published in Haematologica is covered by copyright. All rights are reserved to the Ferrata Storti Foundation. Use of published material is allowed under the following terms and conditions:

https://creativecommons.org/licenses/by-nc/4.0/legalcode. Copies of published material are allowed for personal or internal use. Sharing published material for non-commercial purposes is subject to the following conditions:

https://creativecommons. org//icenses/by-nc/4.0/legalcode, sect. 3. Reproducing and sharing published material for commercial purposes is not allowed without permission in writing from the publisher. 
NADPH oxidases (NOX) as important sources of ROS in platelets responsible for the regulation of platelet responsiveness. ${ }^{9-15}$ Despite the increased interest in this aspect of platelet biology and hemostasis regulation, progress within this field is hampered by the lack of reliable and quantitative techniques for the analysis of platelet oxidative status. ${ }^{16,17}$ This has made it challenging to completely appreciate the importance of endogenous and exogenous oxidants on the regulation of platelet signaling pathways and on the balance between hemostasis and thrombosis in health and disease. Indirectly, this has impeded the development of pharmacological treatments for thrombotic conditions based on the control of ROS generation. We addressed this biomedical need by combining the measurement of platelet activation (using turbidimetry ${ }^{18}$ ) and the simultaneous measurement of intracellular or extracellular oxygen radicals [using electron paramagnetic resonance (EPR) or EPR spectroscopy ${ }^{19,20}$ into one multiplex technique that allows the accurate study of the oxidative status and function of human platelets.

This technique is likely to find application in clinical practice, where the simultaneous analysis of platelet responsiveness and oxidative stress can help develop more advanced diagnostics for patients at risk of thrombotic diseases. It could also find application in drug discovery, as NOX modulation is becoming an important therapeutic strategy in several diseases. ${ }^{21,22}$ In the cardiovascular field, in order to avoid side effects and bleeding complications of antithrombotic drugs, modern drug discovery aims to develop targeted approaches that interfere with the contribution of platelets to pathological alterations of the vascular system while preserving their vascular protective functions. ${ }^{23,24}$ Within this context, it is imperative to deepen our understanding of the regulation of platelets in both health and disease, as redox-dependent regulation of platelets remains poorly understood.$^{25}$ Our novel approach can help to clarify redox-dependent mechanisms regulating platelets and hemostasis, validate new drug discovery targets, and identify novel antiplatelet drug candidates.

In this study, we utilized the EPR/turbidimetry technique to clarify the dynamics of the generation and activation of oxygen radicals in human platelets in response to physiological and pathological stimuli. The use of NOX1- and NOX2-selective peptide inhibitors allowed the identification of key differences in the involvement of these two enzymes in the response to platelet agonists and modulators. The application of this technique will further our understanding of redox-dependent platelet regulation and may have important consequences for antiplatelet drug discovery, where the quest for truly pathway-specific inhibitors targeting pathological platelet activation without interfering with their physiological hemostatic function remains an unmet objective.

\section{Methods}

\section{Platelet preparation}

Human blood was drawn from healthy volunteers by median cubital vein venepuncture following Royal Devon and Exeter NHS Foundation Trust Code of Ethics and Research Conduct and under National Research Ethics Service South West - Central Bristol approval (Rec. n. 14/SW/1089). Sodium citrate was used as anticoagulant $(0.5 \% \mathrm{w} / \mathrm{v})$. Platelet rich plasma (PRP) was separated from whole blood by centrifugation [250xg, 17 minutes (min)], and platelets were separated from PRP by a second centrifugation step (500xg, $10 \mathrm{~min}$ ), in the presence of prostaglandin E1 (PGE1, 40 $\mathrm{ng} / \mathrm{mL}$ ) and indomethacin $(10 \mu \mathrm{M})$. For mouse platelets, blood was taken with intracardiac puncture from 12 -week old females under the Home Office license PPL30/3348 and anticoagulated with $0.5 \% \mathrm{w} / \mathrm{v}$ citrate. PRP was separated from whole blood by centrifugation (160xg, $15 \mathrm{~min}$ ), and platelets were separated from PRP by a second centrifugation step (600xg, $10 \mathrm{~min})$, in the presence of prostaglandin E1 (PGE1, $40 \mathrm{ng} / \mathrm{mL}$ ) and indomethacin $(10 \mu \mathrm{M})$.

\section{Electron paramagnetic resonance/turbidimetry assay}

$2 \times 10^{8}$ platelets $/ \mathrm{mL}$ were prepared as described above. Before adding stimuli, $200 \mu \mathrm{M}$ CMH or PPH was added to platelets in the presence of $25 \mu \mathrm{M}$ deferroxamine and $5 \mu \mathrm{M}$ diethyldithiocarbamate (DETC). Platelet suspensions were loaded onto a Chronolog 700-2 aggregometer with continuous stirring and the turbidimetry readings were immediately started. $50 \mu \mathrm{L}$ of platelet-free supernatant were transferred into the Hirschmann precision micropipettes and read using an e-scan (Noxygen, Germany).

\section{Thrombus formation under physiological flow assay}

The Bioflux200 system (Fluxion, South San Francisco, CA, USA) was used to analyze thrombus formation in human and mouse whole blood under flow. Heparin-anticoagulated whole blood was anticoagulated with $5 \mu / \mathrm{mL}$ heparin plus $40 \quad \mu \mathrm{M}$ D-Phenylalanyl-prolyl-arginyl Chloromethyl Ketone or PPACK and incubated with scrambled or the NOX inhibitory peptides, NoxA1ds and Nox2ds-tat before the addition of $1 \mu \mathrm{M} 3,3$ '-dihexyloxacarbocyanine iodide (DiOC6) for 10 min before the blood was added to the wells. Thrombus formation was visualized by fluorescence microscopy at a shear rate of $1000 \mathrm{sec}^{-1}$.

A more detailed description of the methods used is available in the Online Supplementary Appendix.

\section{Results}

\section{Superoxide anion-dependence of platelet activation by collagen but not thrombin}

Generation of ROS in a living cell can be examined by EPR spectroscopy using 1-hydroxy-3-methoxycarbonyl2,2,5,5-tetramethylpyrrolidine (CMH), an oxygen radicalspecific spin probe. ${ }^{16}$ This spin probe crosses the plasma membrane and directly reacts with intracellular oxygen radicals to generate a nitroxide adduct that can be detected by EPR spectroscopy (Online Supplementary Figure S1A and B). We combined classical aggregometry (also known as turbidimetry) with CMH-dependent and EPR spectroscopy by analyzing CMH oxidation in the platelet supernatant while the aggregation reaction is taking place (Online Supplementary Figure S1C). EPR is considered a gold standard for ROS detection and offers the important advantage of providing a quantification of the generation rate of oxygen radicals. It is in fact possible to build a calibration curve using known concentrations of the nitroxide adduct $\left(\mathrm{CM}^{*}\right)$ (Online Supplementary Figure S2A and B). Once platelet suspension density and incubation time is known, it is possible to interpolate experimental data of resonance intensity to determine $\mathrm{CMH}$ oxidation rates (moles per platelet per min; see formula in Online Supplementary Figure S2C). This assay allowed us to correlate platelet aggregation induced by collagen and thrombin with the rate of oxygen radical generation (measured as rate of $\mathrm{CMH}$ oxidation). Collagen 
was tested at concentrations ranging from 1 to $30 \mu \mathrm{g} / \mathrm{mL}$ and both aggregation kinetics (Figure $1 \mathrm{~A}$ ) and oxygen radical generation rates at $10 \mathrm{~min}$ from stimulus delivery (Figure 1C) were concentration dependent. Similarly to collagen, a synthetic collagen-related peptide (CRP) that selectively engages GPVI receptor (but not other collagen-binding receptors on platelets), also led to concentration-dependent $\mathrm{O} 2^{*-}$ formation that was necessary for platelet aggregation
(Online Supplementary Figure S3). Thrombin also showed concentration dependence of both EPR (Figure 1B) and aggregation (Figure 1D) responses between 0.03 and 1 unit $/ \mathrm{mL}$. Online Supplementary Table S1 shows the EC50 values of these three agonists for EPR detection of superoxide anion generation and aggregation response.

In order to confirm the chemical nature of the oxygen radicals generated by platelets upon activation and detected

\section{A Collagen, EPR}
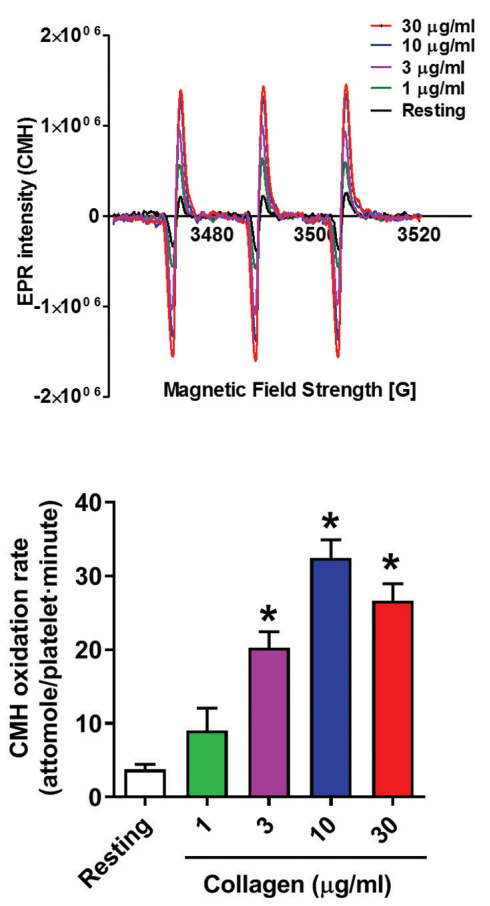

C Collagen, Aggregometry
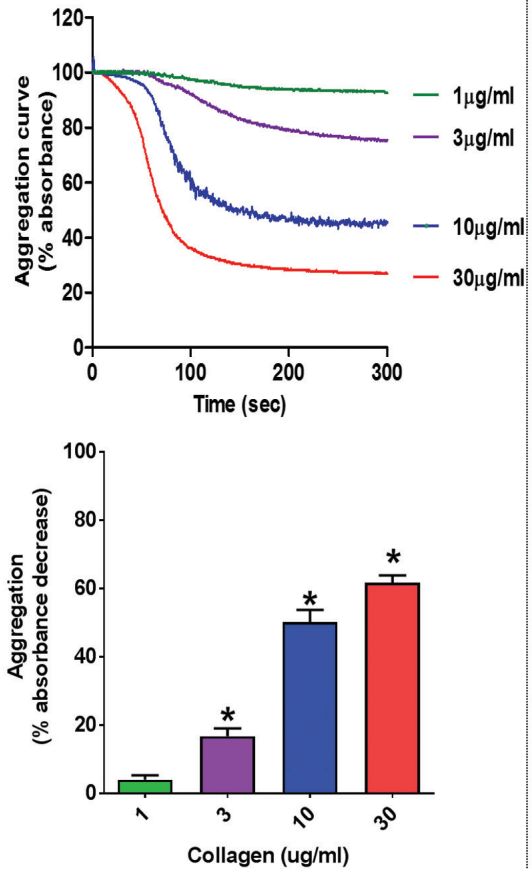

B Thrombin, EPR
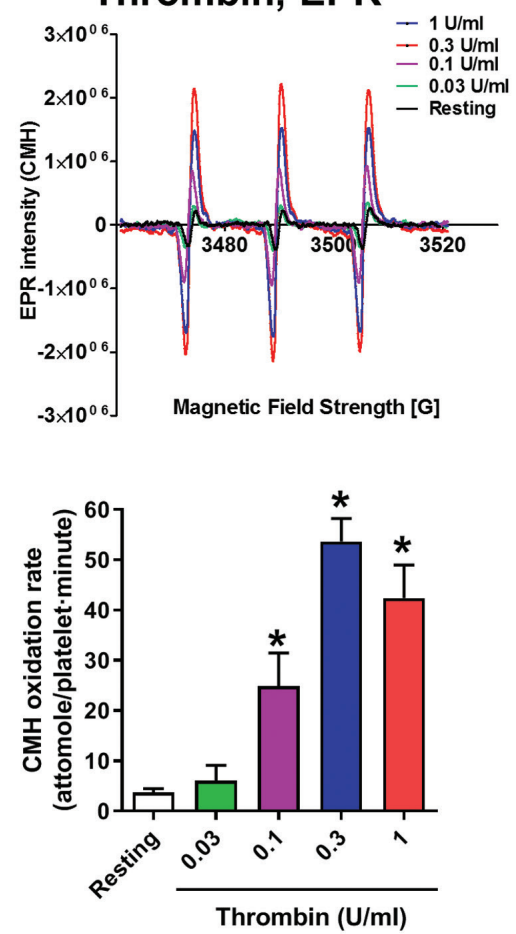

D Thrombin, Aggregometry
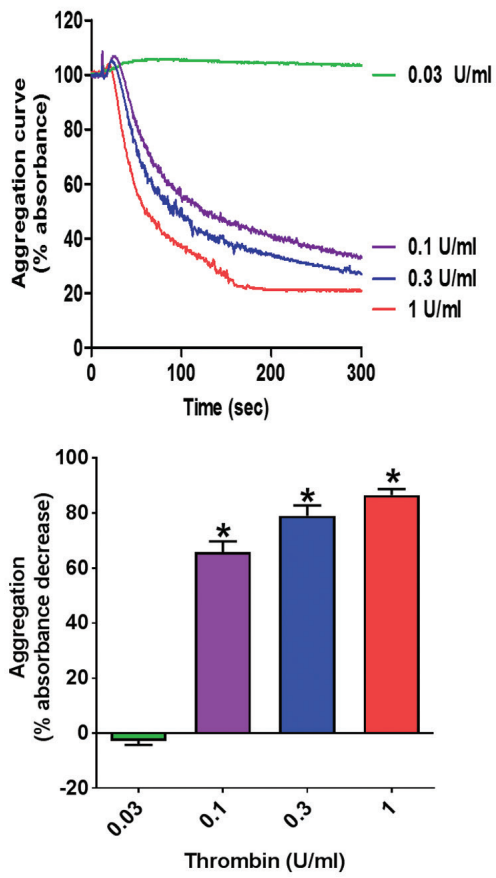

Figure 1. Oxygen radical generation by platelets activated by physiological stimuli collagen and thrombin. Washed human platelets were prepared as described. Collagen was tested at concentrations ranging from 1 to 30 $\mu \mathrm{g} / \mathrm{mL}$ (A and $\mathrm{C}$ ), while thrombin was tested from 0.03 to 1 unit/mL (B and D). Electron paramagnetic resonance (EPR) was used to measure oxygen radicals formation as 1 hydroxy-3-methoxycarbonyl-2,2,5,5-tetramethylpyrrolidine $(\mathrm{CMH})$ oxidation rates [32.7 and 37.3 attomoles per platelet per minute (min), respectively] (A and $\mathrm{C}$ ) and aggregation was simultaneously assessed by turbidimetry $(B$ and $D)$. For either technique, representative examples are shown in top panels, while quantification is shown in the bottom panels. Aggregation curves up to $5 \mathrm{~min}$ are shown, while EPR resonance readings were taken after 10 min of stimulation. Examples of EPR traces and aggregation curves are representative of 4 independent experiments. Statistical analysis was performed by one-way ANOVA with Bonferroni post-hoc test. $* P<0.05$ compared to resting platelets. $\mathrm{N}=4$ for $(A-D)$. 
by EPR, we utilized cell-permeant pegylated superoxide dismutase (PEG-SOD), which scavenges O2*- by dismuting it into H202. The EPR signal detected in human platelets stimulated by collagen and thrombin was abolished by PEG-SOD, suggesting that $\mathrm{O}^{*-}$ is the oxygen radical species generated under these conditions (Online Supplementary Figure S4A and C, respectively). The generation of $\mathrm{O}^{--}$radicals appeared necessary for platelet activation by collagen, as the scavenging of this oxygen radical by PEG-SOD significantly inhibited aggregation in response to this agonist (Online Supplementary Figure S4B). On the other hand, thrombin-dependent aggregation was not affected by PEG-SOD, suggesting that O2- is not an essential component of the signaling cascades induced by this agonist. Similarly to collagen, both EPR and aggregation signals in response to CRP were abolished by PEGSOD (Online Supplementary Figure S3C and D). The data obtained with PEG-SOD were confirmed using another O2*- scavenger, 4-hydroxy-2,2,6,6-tetramethylpiperidin-1oxyl (or TEMPOL). TEMPOL abolishes the EPR signal in response to either collagen or thrombin (Online Supplementary Figure S5A and C, respectively), while only the aggregation response to collagen but not thrombin (Online Supplementary Figure S5C and D, respectively) is inhibited. Interestingly, the non-selective antioxidant and cell permeable amino acid $\mathrm{N}$-acetyl cysteine (NAC) inhibits aggregation induced by both collagen and thrombin, suggesting that the thrombin responses depend on oxidative reactions, but that $\mathrm{O}^{--}$, per se, is not the oxidant species involved in this response (Online Supplementary Figure S5C and $D$ ). In order to confirm that the CMH-based EPR did not detect $\mathrm{H}_{2} \mathrm{O}_{2}$ (another major ROS) in our hands, we performed experiments in the presence of catalase (CAT, noncell permeable) and pegylated catalase (PEG-CAT, cell permeable), which convert $\mathrm{H} 2 \mathrm{O2}$ to water (Online Supplementary Figure S6). No effect of either enzymes on the collagen- or thrombin-induced EPR signal was observed, proving the specificity of the CMH-based EPR measurements for oxygen radicals and $\mathrm{O}^{--}$in particular.

\section{Low levels of superoxide anion are released by platelets although they are not required for platelet activation}

We demonstrated that the CMH-based EPR spectroscopy predominantly measures intracellular but not extracellular platelet $\mathrm{O}^{-}$in response to either collagen or thrombin (Online Supplementary Figure S7), as the non-pegylated and thus non-cell permeable version of the enzyme superoxide dismutase (SOD) did not affect the responses. Interestingly, we also utilized a non-cell permeable EPR probe called 1Hydroxy-4-phosphono-oxy-2,2,6,6-tetramethylpiperidine (or $\mathrm{PPH}$ ) to detect extracellular $\mathrm{O}^{--}$released by platelets (Online Supplementary Figure S8A-D). Noticeably, the scavenging of extracellular $\mathrm{O}^{*-}$ with $\mathrm{SOD}$ was effective in abolishing the PPH-based signal (Online Supplementary Figure $S 8 E$ and $F$ ), but did not affect collagen- or thrombin-induced aggregation (Online Supplementary Figure S8G and H), suggesting that extracellular $\mathrm{O} 2$ - does not participate in the process of platelet activation. In addition, we confirmed the formation of $\mathrm{H}_{2} \mathrm{O}_{2}$ in response to collagen or thrombin using the $\mathrm{H}_{2} \mathrm{O}_{2}$-specific probe Amplex Red (Online Supplementary Figure $S 9 A$ and $B$ ) and that catalase effectively quenched the signal. More importantly, the degradation of $\mathrm{H}_{2} \mathrm{O}_{2}$ by catalase or pegylated catalase inhibited platelet aggregation stimulated by thrombin, but not collagen (Online Supplementary Figure $S 9 C-F$ ). This suggests that $\mathrm{H}_{2} \mathrm{O}_{2}$ is a crit- ical component of the signaling triggered by thrombin but not collagen.

\section{Differential role of NADPH oxidases 1 and 2 in collagen- and thrombin-dependent activation of human platelets}

We utilized the combinatorial EPR/aggregometry that we developed to assess the role of NOX1 and NOX2 in platelet activation by collagen and thrombin. In experiments illustrated in Figure 2, we used the NOX1-specific inhibitory peptide NoxA1ds to assess the role of this enzyme in platelet activation. ${ }^{26}$ NOX1 inhibition almost completely inhibited oxygen radical formation in response to collagen (Figure 2A), but not thrombin (Figure 2B). In parallel, collagen- (Figure 2C) but not thrombin-dependent (Figure 2D) aggregation was inhibited by NoxA1ds. Importantly a scrambled version of the peptide was used as control. Taken together, these data suggest that NOX1 is activated and participates in the signaling of platelet activation in response to collagen but not thrombin. We also tested the role of NOX2 using the specific inhibitory peptide Nox2ds-tat. ${ }^{27,28}$ Both collagen- and thrombindependent oxygen radical formation are significantly impaired by NOX2 inhibition (Figure 3A and B, respectively). Interestingly, although the inhibition of the thrombin response by Nox2ds-tat reduces oxygen radical formation to basal levels, the inhibition of collagen-induced oxygen radical levels is only partial (i.e. in the presence of Nox2ds-tat the oxygen radical levels induced by collagen are significantly higher than resting controls). This is reflected in the aggregation data, which show complete inhibition by Nox2ds-tat of the aggregation induced by thrombin but only marginal inhibition of the response to collagen (Figure 3D and C, respectively). This is consistent with NOX1 playing a larger role than NOX2 in collageninduced platelet aggregation. The data obtained with collagen were essentially confirmed using the synthetic ligand for GPVI CRP (Online Supplementary Figure S10), which suggests that GPVI is the key receptor linking collagen-dependent platelet activation to oxygen radical generation and determines the redox patterns triggered by collagen in platelets. The active engagement of NOX1 and NOX2 in collagen and thrombin signaling was confirmed by co-immunoprecipitation of these two core subunits with respective essential cytosolic components of each complex. NOX1 is co-immunoprecipitated with its canonical activating subunit NOXA129 in the presence of collagen, which is consistent with NOX1 being post-translationally activated in response to collagen (Figure 4A). NOX2 appears to be co-immunoprecipitated with its canonical organizing subunit $\mathrm{p} 47 \mathrm{phox}$ in the presence of thrombin and weakly in the presence of collagen (Figure 4B). This aligns with the conclusions reached using our EPR and aggregation experiments, i.e. that collagen activates a primarily NOX1-dependent response, while thrombin activates primarily NOX2. Accordingly, whole blood thrombus formation experiments on collagen showed that NOX1 inhibition with NoxA1ds abolishes thrombus formation (Figure 4C), while NOX2 inhibition by Nox2ds-tat induces only a marginal inhibition (Figure 4D). Experiments in platelets from wild-type, NOX1" or NOX2 ${ }^{-r}$ mice confirmed the centrality of NOX1 for collagen signaling with marginal involvement of NOX2 in the aggregation response (Figure 5A and B), while NOX2 is critical for thrombin signaling (Figure 5C). 
Role of NOX1 and NOX2 in mediating the effect on platelets of weak agonists/positive modulators oxidized LDL (oxLDL) and amyloid beta (AB1-42)

Using these tools (NoxA1ds ${ }^{26}$ and Nox $2 \mathrm{ds}^{-\mathrm{tat}^{27,28}}$ ), we determined that NOX1 or NOX2 play an equivalent role in the generation of superoxide anion in response to oxLDL (Figure 6A) and that both enzymes are required for the stimulation of the modest aggregation induced by this modulator (Figure 6B). As suggested by the inhibition of aggregation by PEG-SOD, $\mathrm{O}^{--}$is the oxidant molecule required for the activation of platelets by oxLDL. It is important to note how oxLDL elicits a very modest aggregation (both as size and kinetic of the response), which conforms to the designation of oxLDL as a modulator rather than agonist (i.e. exerting its effect by enhancing the responsiveness of platelets to low agonist levels). In fact, low concentrations of collagen (Figure $6 \mathrm{C}$ ) or thrombin (Figure 6D) characterized by the ability to induce no/low aggregation, resulted able to induce a robust platelet aggregation in the presence of ox-LDL. The synergistic effect of oxLDL in combination with thrombin or collagen was inhibited by NoxA1ds (Figure 6C) or Nox2ds-tat (Figure $6 \mathrm{D})$, respectively. We could not test NoxA1ds on ox-LDL + collagen or Nox2ds-tat on oxLDL + thrombin, because, as shown above, NoxA1ds inhibits collagen directly and Nox2ds-tat inhibits thrombin directly. Similarly, A $\beta 1-42$ induces $\mathrm{O}^{--}$formation via activation of NOX1 and NOX2 (Figure 7A), which leads to a modest platelet aggregation
A Collagen + NOX1 inhibitor, EPR

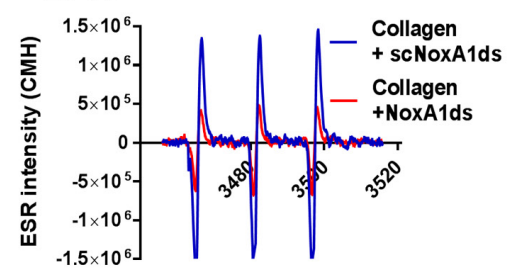

Magnetic Field Strength [G]

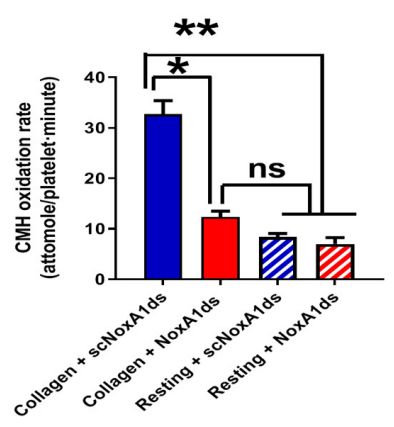

C Collagen + NOX1 inhibitor, Aggregometry
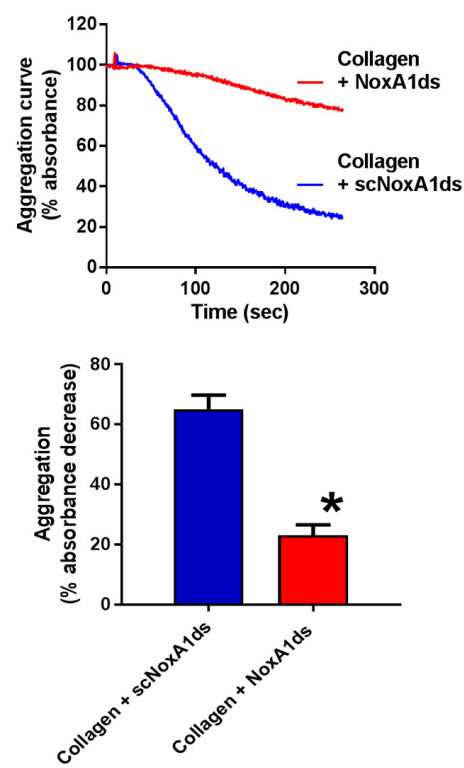

B Thrombin + NOX1 inhibitor, EPR

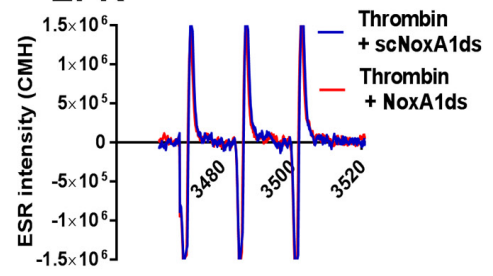

Magnetic Field Strength [G]

**

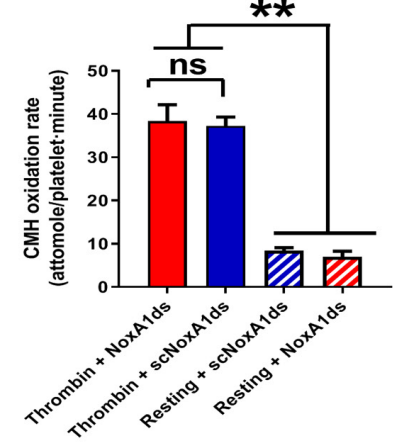

D Thrombin + NOX1 inhibitor, Aggregometry
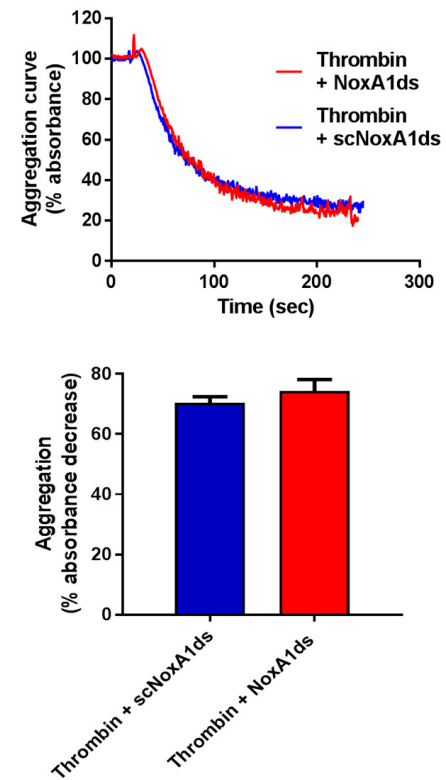

Figure 2. NOX1 is specifically involved in platelet activation by collagen but not thrombin. 1-hydroxy-3-methoxycarbonyl2,2,5,5-tetramethylpyrrolidine $(\mathrm{CMH})$ was utilized for the detection of oxygen radicals generated by platelets. $10 \mu \mathrm{g} / \mathrm{mL}$ collagen (A) or 0.1 unit/mL thrombin (B) were tested. $10 \mu \mathrm{M}$ of NoxA1ds abolished the electron paramagnetic resonance (EPR) response measured in the presence of collagen. The scrambled peptide at the same concentration (scNoxA1ds) was used as a negative control. Interestingly, the inhibition of NOX1 by NoxA1ds also inhibited collagen-dependent (C), but not thrombin-dependent (D) platelet aggregation. Aggregation curves for up to 5 minutes ( $\mathrm{min}$ ) are shown, while EPR resonance readings were taken after $10 \mathrm{~min}$ of stimulation. Examples of EPR traces and aggregation curves are representative of 3 or more independent experiments. Statistical analysis was performed by one-way ANOVA with Bonferroni post-hoc test for EPR. $* P<0.05$ compared to resting platelets or $t$-test for aggregation. $\star P<0.05$ compared to scrambled control. $\mathrm{N}=3$ for $(A-D)$. 
(Figure 7B). On the other hand, similarly to oxLDL, A $\beta 1-42$ displays the ability to synergistically increase the aggregation response to low concentrations of collagen (Figure 7C) or thrombin (Figure 7D). As proved with inhibitory peptides NoxA1ds26 and Nox2ds-tat, ${ }^{27,28}$ the synergistic effect on collagen- or thrombin-induced aggregation is NOX1- or NOX2-dependent, respectively. We could not test NoxA1ds on A $\beta 1-42$ + collagen or Nox2ds-tat on A $\beta 1-42$ + thrombin, because, as shown above, NoxA1ds inhibits collagen directly and Nox2ds-tat inhibits thrombin directly. Transgenic mice $\mathrm{NOX}^{-1}$ and $\mathrm{NOX}_{2}{ }^{-1}$ were utilized to assess the NOXdependence of the responses to oxLDL and A $\beta 1-42$. As oxLDL coating of surfaces is not commonly used and there is no accepted protocol for this procedure, we tested the effect of oxLDL added to mouse blood on thrombus formation on low levels (i.e. $0.05 \mathrm{mg} / \mathrm{mL}$ ) of collagen coating (Figure 8A). These data showed that oxLDL potentiates the thrombus formation on collagen in a NOX2-dependent manner (as it was not evident in $\mathrm{NOX}^{-}$blood). NOX1 ablation inhibits collagen responses directly, therefore we could not investigate the role of NOX1 in the potentiation of responses to this agonist by oxLDL. The ablation of either NOX1 or NOX2 silencing significantly also impairs thrombus formation at physiological arterial shear on absorbed A $\beta 1-42$ (1,000 sec-1) (Figure 8C). At low shear (200 sec-1), mouse platelets display low levels of adhesion to absorbed A $\beta 1-42$ without formation of thrombi, which was inhibited by NOX1 genetic silencing but unaffected by
A

NOX1 immunoprecipitation

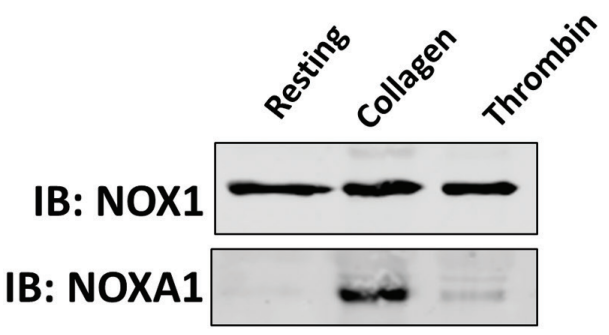

C

Collagen + NOX1 inhibitor, Flow assay
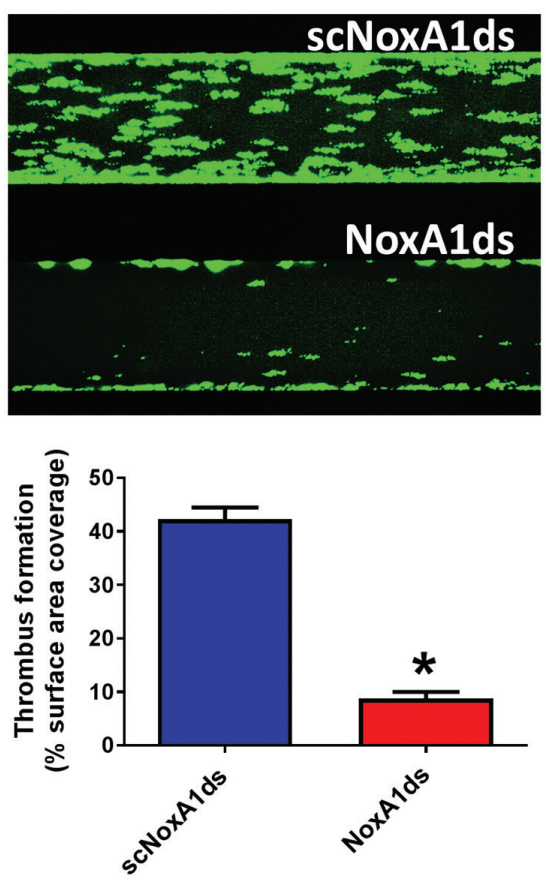

B

\section{NOX2 immunoprecipitation}

IB: NOX2

IB: p47phox

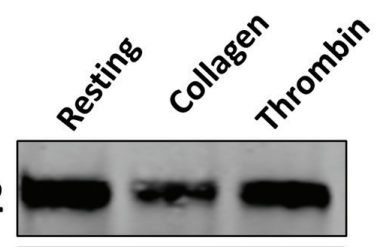

D

\section{Collagen + NOX2 inhibitor, Flow assay}
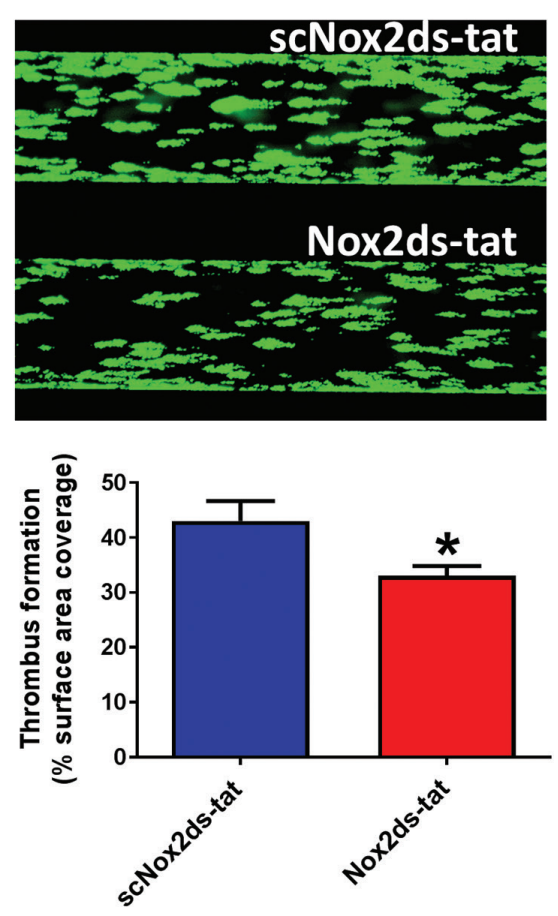

Figure 3 . NOX2 is activated by both collagen and thrombin, but essential only for platelet aggregation induced by thrombin. 1 hydroxy-3-methoxycarbonyl2,2,5,5-tetramethylpyrrolidine (CMH) was utilized for the detection of oxygen radicals generated by platelets. $10 \mu \mathrm{g} / \mathrm{mL}$ collagen (A) or 0.1 unit/mL thrombin (B) were tested. $10 \mu \mathrm{M}$ of Nox2ds-tat inhibited the electron paramag netic resonance (EPR) response measured in the presence of either collagen or thrombin, although the collagen-dependent response remained significantly higher than resting levels of oxygen radical formation. The scrambled peptide at the same concentration (scNox2ds-tat) was used as a negative control. Interestingly, the inhibition of NOX2 by Nox2ds-tat also inhibited thrombin-dependent (D), but not collagen-dependent (C) platelet aggregation. Examples of EPR traces and aggregation curves are representative of 3 or more independent experiments. Statistical analysis was performed by one-way ANOVA with Bonferroni post-hoc test for EPR. $* P<0.05$ compared to resting platelets or $t$-test for aggregation. $\star P<0.05$ compared to scrambled control. $N=4$ for (B and $D), n=5$ for $(A)$, and $n=7$ for $(C)$. 
NOX2 ablation (Online Supplementary Figure S11). As these modulators induce little or no aggregation on their own, we analyzed the modulatory effect of oxLDL and A $\beta 1-42$ in aggregation experiments using $\mathrm{NOX}^{-/}$and $\mathrm{NOX} 2^{-/}$mouse platelets. We showed that oxLDL (Figure 8B) and A $\beta 1-42$
(Figure 8D) potentiate the aggregation induced by collagen in wild-type mice but not in NOX2 ${ }^{-/}$mice, and the aggregation stimulated by thrombin in wild-type mice but not in $\mathrm{NOX}^{-/}$mice. The potentiation of collagen response could not be tested in $\mathrm{NOX}^{-/}$mice, which do not respond to this

\section{A NOX1 immunoprecipitation}

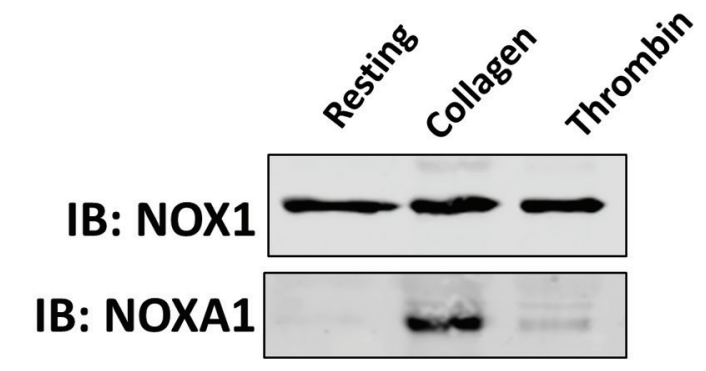

C Collagen + NOX1 inhibitor, Flow assay
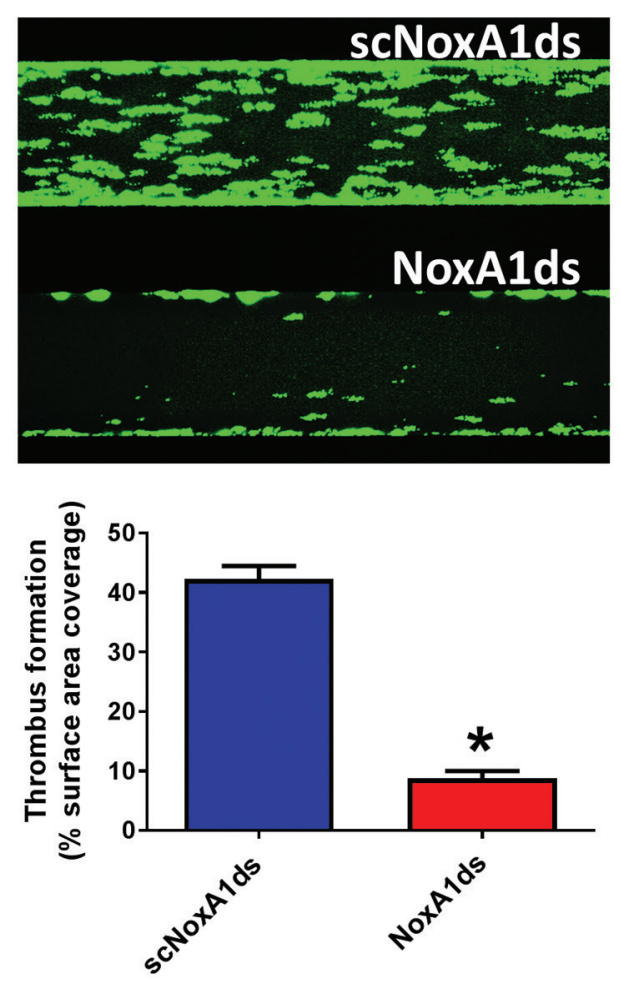

B NOX2 immunoprecipitation

\section{Collagen + NOX2 inhibitor,} Flow assay
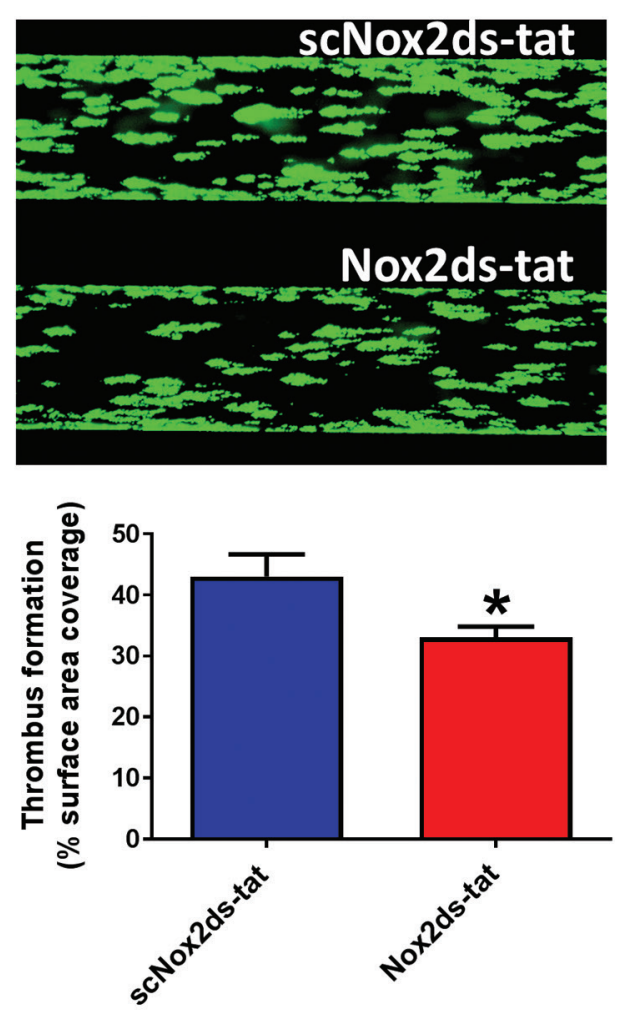

Figure 4. NOX1 is the main source of superoxide anions in human platelet response to collagen, while NOX2 is the main source of thrombin-dependent reactive oxygen species (ROS). The activation of NOX1 (A) and NOX2 (B) was assessed by co-immunoprecipitation with their canonical activating and organizing subunits NOXA1 and p $47^{\text {phox }}$, respectively. $400 \mu \mathrm{L}$ of platelet suspension $\left(4 \times 10^{8}\right.$ platelets $\left./ \mathrm{mL}\right)$ were stimulated with $10 \mu \mathrm{g} / \mathrm{mL}$ collagen or 0.1 unit $/ \mathrm{mL}$ thrombin or vehicle solution (Tyrode's buffer) for 10 minutes ( $\mathrm{min}$ ) before gentle cell lysis (NP4O buffer). Specific NOX1 or NOX2 antibodies and Protein A/G were used to immunoprecipitate the NOX complexes (which by extension should include their regulatory subunits after activation). The immunoprecipitates were tested by immunoblotting using NOX1, NOXA1, NOX2 or p47phox antibodies (as indicated). The data are representative of 4 independent experiments. The functional role of NOX1 and NOX2 in collagen-dependent platelet activation was assessed in a whole blood flow assay ( $C$ and D). Platelets were stained with DiOC6 as described and the Bioflux platform (Fluxion, San Francisco, CA, USA) was utilized to assess the thrombus formation induced by collagen under physiological flow (1000 sec-1). The experiments were performed in the presence of NoxA1ds or its negative scrambled control (C) or Nox2ds-tat or its negative scrambled control (D). Images were taken at 10 min of flow and are representative of 4 independent experiments. They were quantified by assessing the surface area coverage by platelets ( $\mathrm{C}$ and D, bottom). Statistical significance was tested by $t$-test. $* P<0.05$ compared to scrambled control. $N=4$ for ( $\mathrm{C}$ and $\mathrm{D}$ ). 
stimulus, while the potentiation of thrombin responses could not be tested in NOX $2^{-\%}$, for the same reason.

\section{Discussion}

The study of platelet function is critical to understanding vascular homeostasis and disease. A thorough understanding of the redox-dependent regulation of platelet function has been hampered by the lack of a reliable technique to measure intracellular ROS. ${ }^{16,17}$ We have resolved this problem by optimizing an EPR-based technique for the detection of oxygen radicals and combining it with a classical turbidimetric assay for the simultaneous measurement of platelet aggregation. Although EPR has previously been used for the analysis of ROS formation in live cells; ${ }^{16,17}$ this technique has never been applied to the study of platelet redox signaling. We utilized the assay developed here to clarify several unknown aspects of the regulation of platelet activity by endogenous oxidants.

The molecular mechanisms underlying the redox dependence of platelet activation in response to collagen, thrombin, and oxidized LDL are summarized in Online Supplementary Figure S12. Although, the dependence of platelet activation on the generation of endogenous ROS has been described, ${ }^{1-6}$ here for the first time we elucidated the chemical nature of the ROS involved in the signaling of different platelet agonists and modulators. $\mathrm{O}^{--}$are generated in response to all tested agonists and modulators, but although these ROS are directly involved in the signaling of collagen, oxLDL and A $\beta 1-42$, their dismutation to hydrogen peroxide is necessary for the signaling of thrombin. The literature on this aspect of platelet biology is quite inconclusive because of the use of different techniques and conditions. Although previous studies reported the generation of hydrogen peroxide in response to thrombin (and leading to
A Collagen
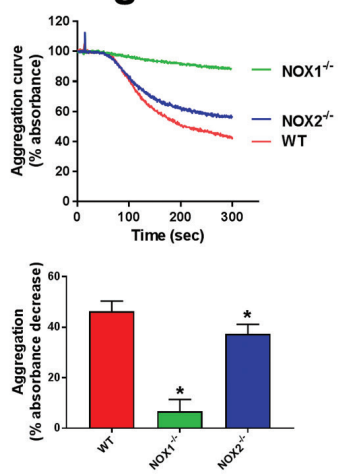

\section{B Collagen}
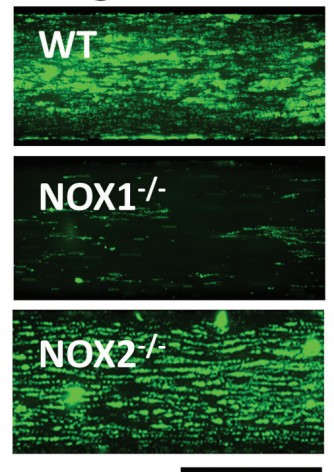

C Thrombin
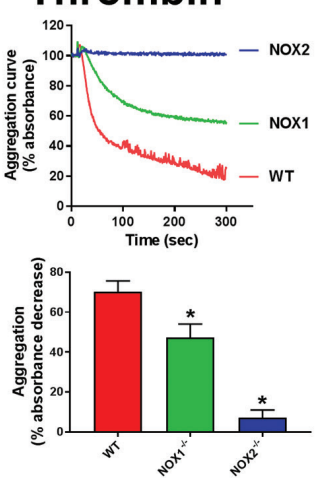
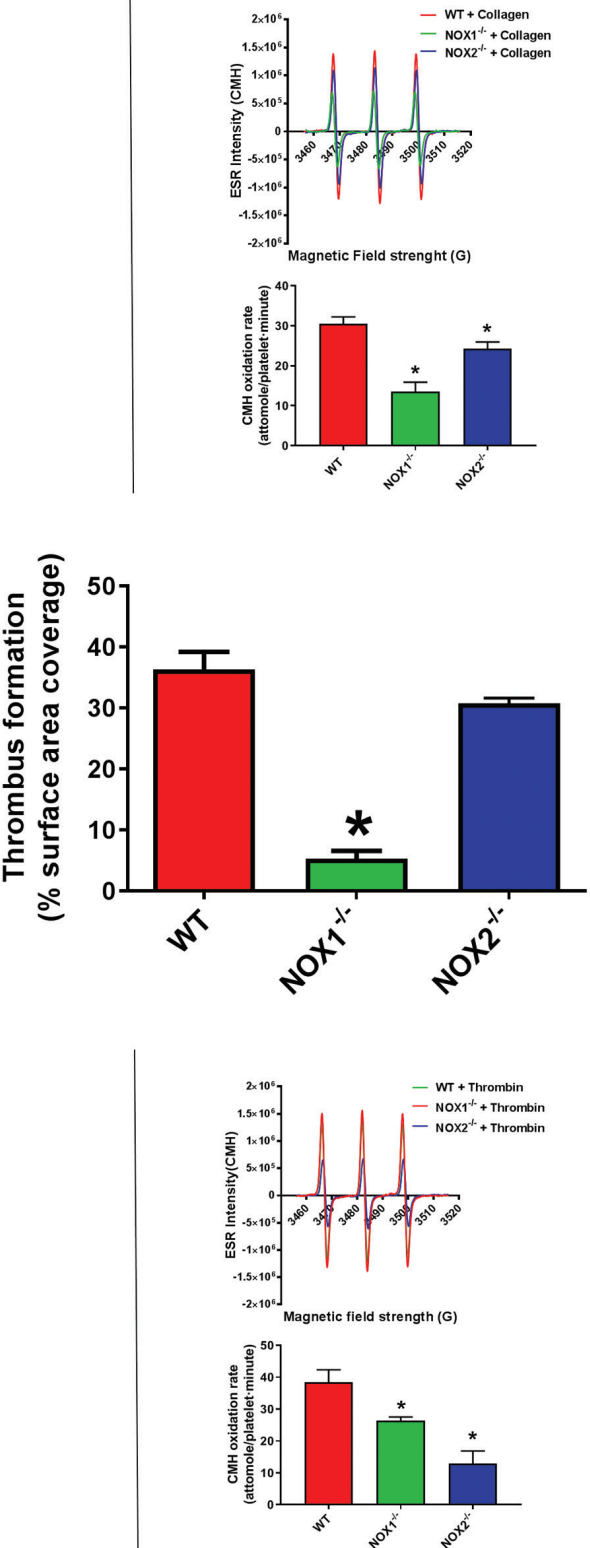

Figure 5. NOX1- and NOX2-dependence of collagen and thrombin aggregation and superoxide generation tested in transgenic mice. Platelets were isolated for wild-type (C57BL6/J), NOX1\% or NOX $\%$ mice exsanguinated via intracardiac puncture and resuspended at $2 \times 10^{8}$ platelets $/ \mathrm{mL}$ density. Platelets were stimulated with $3 \mu \mathrm{g} / \mathrm{mL}$ collagen (A) or 0.1 unit/mL thrombin (C). Aggregation (left) and superoxide anion formation (right) were measured as described for 5 and 10 minutes ( $\mathrm{min}$ ) respectively. (B) The functional role of NOX1 and NOX2 in collagen-dependent platelet activation was also assessed in a whole blood flow assay. Platelets were stained with DiOC6 and the Bioflux platform (Fluxion, San Francisco, CA, USA) was utilized to assess the thrombus formation induced by collagen under physiological flow (1,000 sec-1). Images were taken at $10 \mathrm{~min}$ of flow and are representative of 4 independent experiments. They were quantified by assessing the surface area coverage by platelets with Image J. Data are representative of 4 independent experiments. Statistical analysis was performed by one-way ANOVA with Bonferroni post-hoc test. ${ }^{*} P<0.05 . \mathrm{N}=4$ for (A-C). 
apoptosis) ${ }^{30}$ this study for the first time highlights a significant difference in the role of hydrogen peroxide in the responses to collagen and thrombin. Our observations on the link between thrombin-dependent activation and hydrogen peroxide contradict previous reports pointing to a role for hydrogen peroxide in collagen but not thrombin signaling. ${ }^{31}$ The experimental differences in our and previ- ous studies are extensive and the poor specificity of the tools used for older studies (e.g. 2',7'-dichlorofluorescein diacetate or DCFDA) are potentially responsible for these discrepancies.

Another important addition to our understanding of redox regulation of platelets is the clarification of whether oxidants act intracellularly or extracellularly. Our data clear-
A
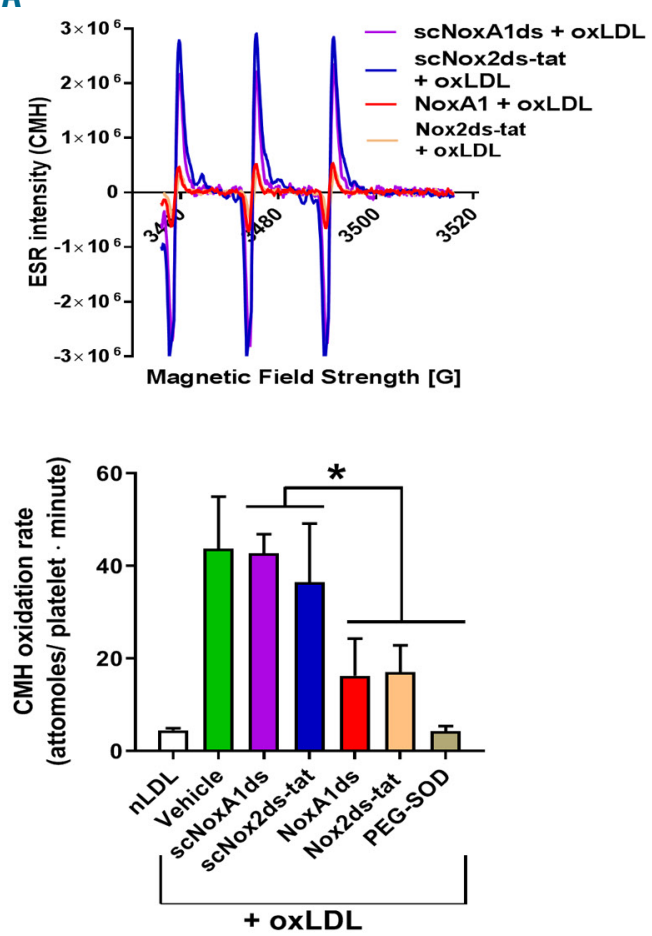

C

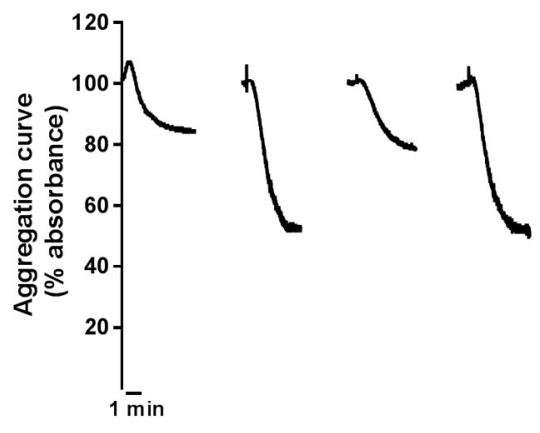

B
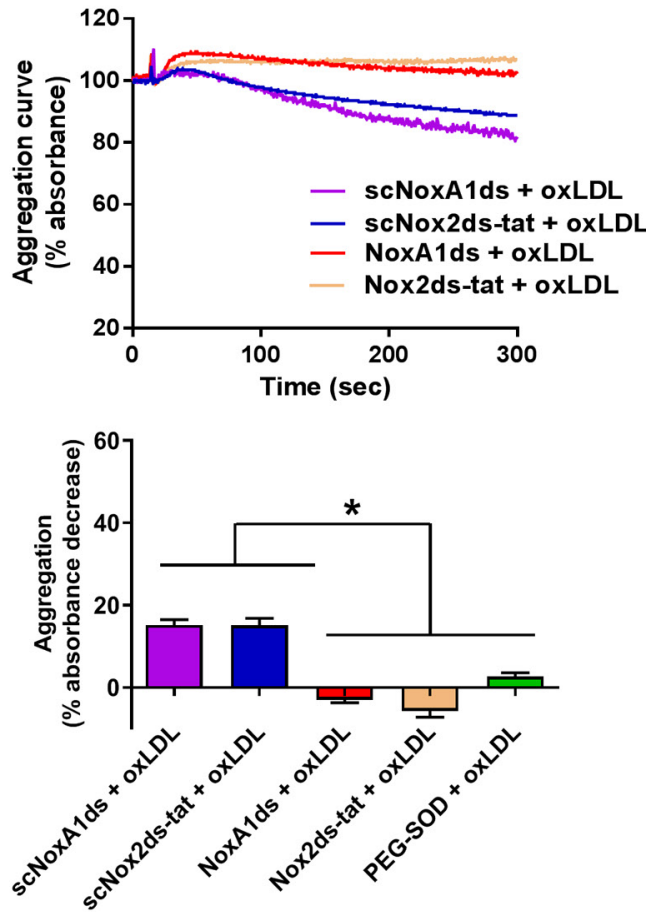

D

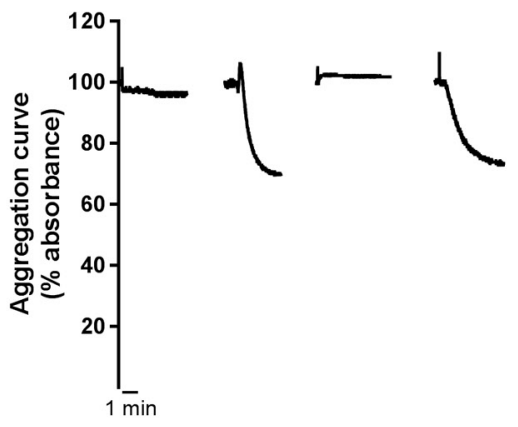

$\begin{array}{lllll}\text { Thrombin } & + & + & + & + \\ \text { nLDL } & + & - & - & - \\ \text { oxLDL } & - & + & + & + \\ \text { NoxA1ds } & - & - & + & - \\ \text { scNoxA1ds } & - & - & - & +\end{array}$

Figure 6. NOX1 and NOX2 are required for the induction of superoxide anion formation and platelets activation by oxidized low density lipoprotein (oxLDL). Oxygen radical generation (A) and platelet aggregation (B) in response to oxLDL were measured as described. 10 uM of scrambled NoxA1ds (scNoxA1ds), NoxA1ds, scrambled Nox2ds-tat (scNox2ds-tat), Nox2ds-tat or 100 unit/mL of PEG-SOD were pre-incubated 10 minutes (min) before platelet stimulation with 50 ng/mL oxLDL. Examples of electron paramagnetic resonance (EPR) traces and aggregation curves are representative of 4 independent experiments. Statistical analysis was performed by one-way ANOVA with Bonferroni post-hoc test. $* P<0.05 . N=3$ for $(B)$ and $n=4$ for $(A)$. The effect of oxLDL as platelet modulator was investigated by traditional aggregometry (C and D). Pre-incubation with $50 \mathrm{ng} / \mathrm{mL}$ oxLDL for $10 \mathrm{~min}$ was followed by stimulation with $3 \mathrm{ug} / \mathrm{mL} \mathrm{collagen}(\mathrm{C})$ or 0.03 unit/mL thrombin (D). In order to test the dependence of the modulatory effect of oxLDL on NOX1 and NOX2, $10 \mu \mathrm{M}$ scrambled NoxA1ds (scNoxA1ds), NoxA1ds, scrambled Nox2ds-tat (scNox2ds-tat), Nox2ds-tat were pre-incubated (10 min before addition of oxLDL). Aggregation curves are representative of 4 independent experiments. 
ly suggest that platelet $\mathrm{O}^{--}$acts intracellularly in these responses, as only cell permeable scavenger can affect their activity (i.e. PEG-SOD), while hydrogen peroxide is likely to be formed by dismutation of intracellular $\mathrm{O} 2^{\circ-}$ and released extracellularly, where it potentiates platelet responses induced by thrombin (as proved by the effect of non-cell permeable catalase shown in Online Supplementary
Figure S6). These findings add significantly to our understanding of platelet redox regulation and are in agreement with previous suggestions of an intracellular function for $\mathrm{O} 2^{--} .^{32,33}$ It is also in agreement with the observation of a positive regulatory role for extracellular hydrogen peroxide in thrombin-induced responses ${ }^{34}$ and a role for extracellular oxidants in the regulation of platelet surface receptor func-
A
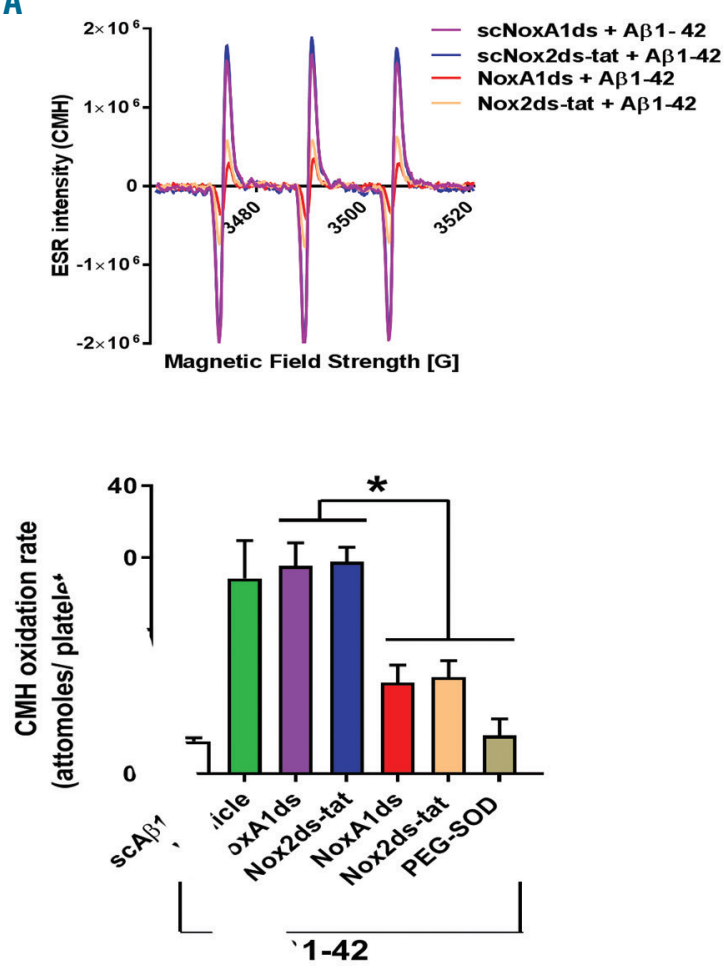

C

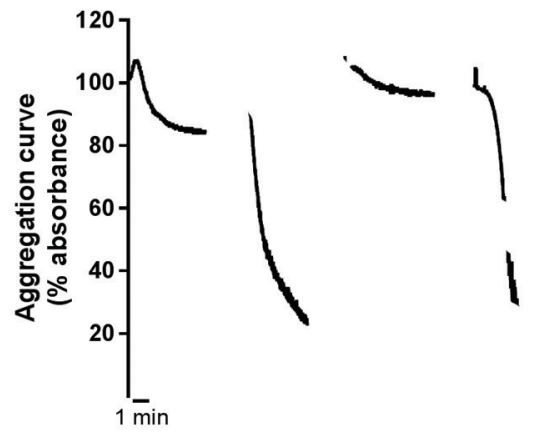

B
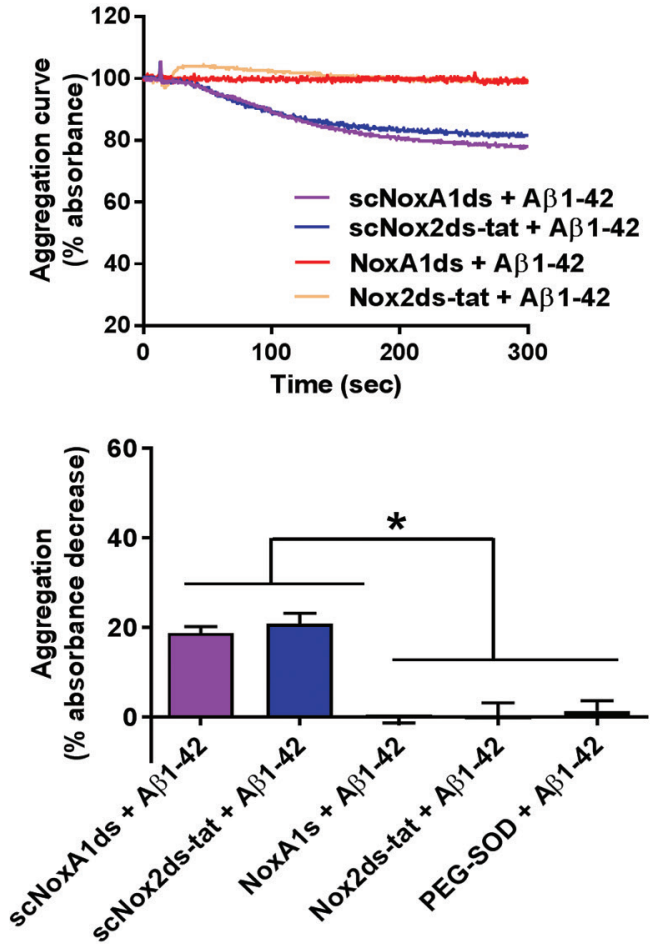

D

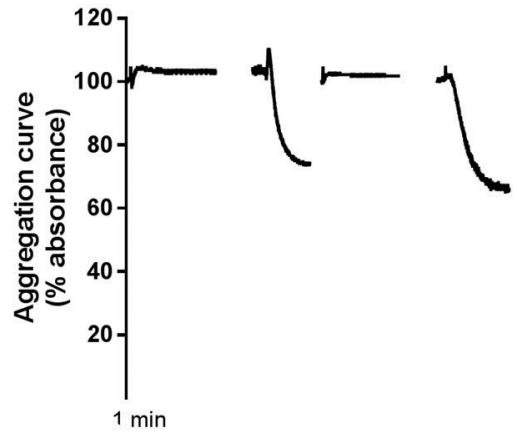

$\begin{array}{lllll}\text { Throm } & & + & + & + \\ \text { ScA } \beta 1-42 & & - & - & - \\ \text { A } \beta 1-42 & & & + & + \\ \text { NoxA1ds } & - & & + & - \\ \text { scNoxA1ds } & - & - & - & +\end{array}$

Figure 7. NOX1 and NOX2 are required for the induction of superoxide anion formation and platelet activation by amyloid peptide $\beta$ 1-42. Oxygen radical generation (A) and platelet aggregation (B) in response to amyloid peptide $\beta$ 1-42 (AB1-42) were measured as described. $10 \mu \mathrm{M}$ of scrambled NoxA1ds (scNoxA1ds), NoxA1ds, scrambled Nox2ds-tat (scNox2ds-tat), Nox2ds-tat or 100 unit/mL of PEG-SOD were pre-incubated 10 minutes (min) before platelet stimulation with $20 \mu \mathrm{M}$ A $\beta 1$-42. Examples of electron paramagnetic resonance (EPR) traces and aggregation curves are representative of 4 independent experiments. Statistical analysis was performed by one-way ANOVA with Bonferroni post-hoc test. $* P<0.05$. $\mathrm{N}=3$ for (A and B). The effect of A 1 1-42 as platelet modulator was investigated by traditional aggregometry (C and D). Pre-incubation with $20 \mu \mathrm{M}$ A $\beta 1-42$ for 10 min was followed by stimulation with $3 \mu \mathrm{g} / \mathrm{mL}$ collagen (C) or $0.03 \mathrm{unit} / \mathrm{mL}$ thrombin (D). In order to test the dependence of the modulatory effect of A $31-42$ on NOX1 and NOX2, $10 \mu \mathrm{M}$ scrambled NoxA1ds (scNoxA1ds), NoxA1ds, scrambled Nox2ds-tat (scNox2dstat), Nox2ds-tat were pre-incubated (10 min before addition of Aß1-42). Aggregation curves are representative of 4 independent experiments. 
tion by protein disulphide isomerases (PDI). ${ }^{35}$ Regarding the redox-dependence of the collagen signaling in platelets, although further studies are required, protein tyrosine phosphatases (PTP) are the most likely link between redox and conventional signaling in platelets. ${ }^{36}$ The oxidative inactivation of protein phosphatases has in fact been suggested to play a key role in the activation and regulation of the pathophysiological roles of platelets. ${ }^{37}$ The Src Homology Phosphatase 2 (SHP2) has been shown to play an important role as a negative regulator of platelet activation, ${ }^{38}$ and recent studies demonstrated that ROS generated during platelet activation oxidize and inhibit SHP2. This, in turns, facilitates the activation of protein kinase-mediated signaling pathways and drives the processes associated with cell activation (e.g. adhesion receptor activation, shape change, etc.). ${ }^{37,39}$

In this study, we also highlight the differential involvement of NOX1 and NOX2 in physiological agonist signaling (i.e. collagen and thrombin). NOX1 is essential for the signaling of collagen with NOX2 inhibition only partially
A
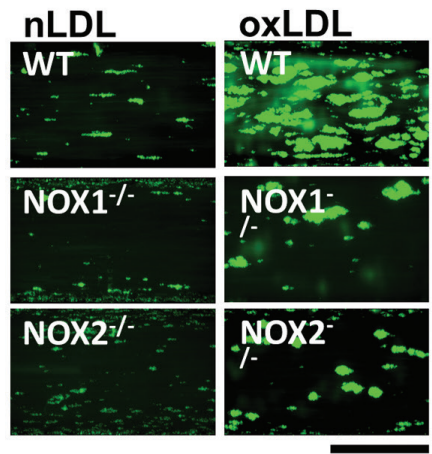

B

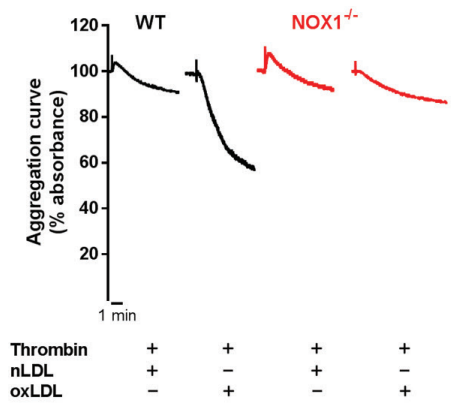

C
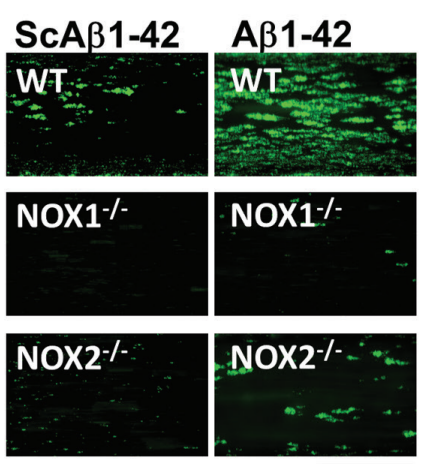

D

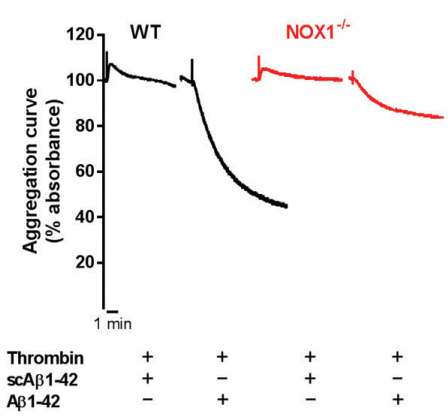

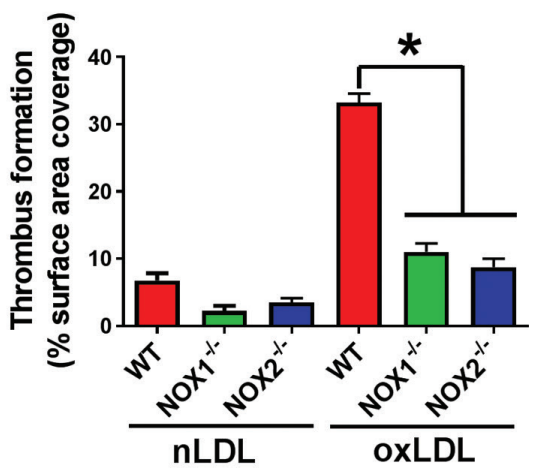
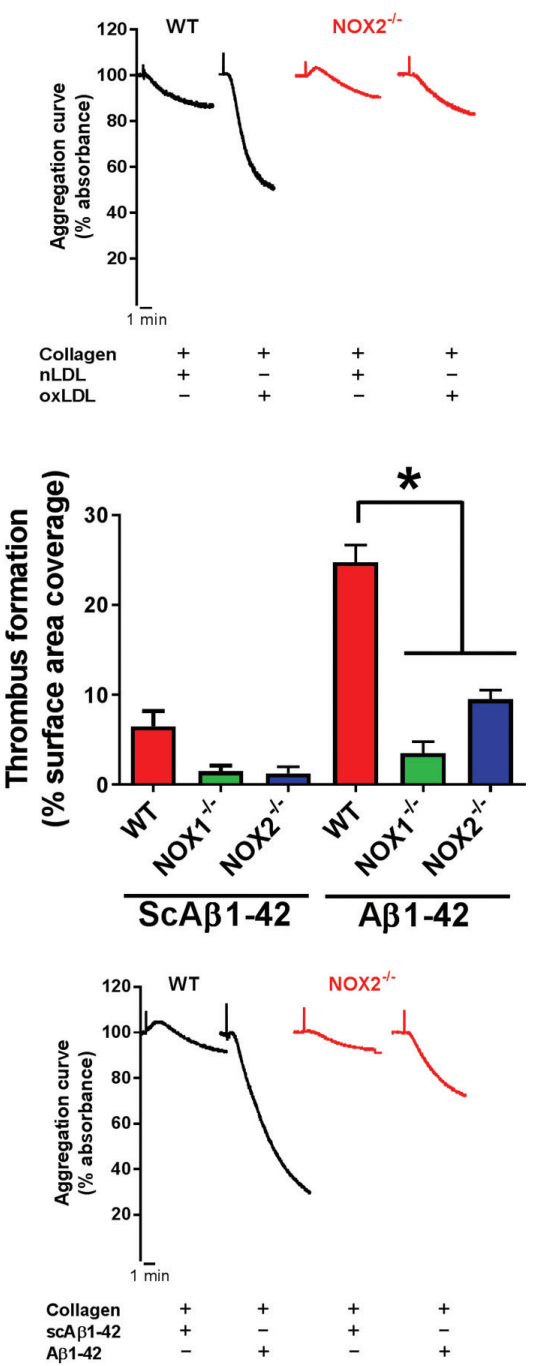

Figure 8. Experiments on transgenic mice suggest that NOX1 and NOX2 are required for the effect of amyloid peptide $\beta$ 142 and oxidized low density lipoprotein (oxLDL). Platelets from wild-type, NOX1\% or NOX2 mice were stained with DiOC6 and the Bioflux platform (Fluxion, San Francisco, CA, USA) was utilized to assess the thrombus formation induced by collagen under physiological flow. (A) Ibidi Vena8 ${ }^{+}$flow chambers were coated with 0.05 $\mathrm{mg} / \mathrm{mL}$ collagen and whole blood was treated with 50 $\mathrm{ng} / \mathrm{mL}$ native LDL ( $\mathrm{nLDL}$ ) or oxLDL. (C) Ibidi Vena8+ flow chambers were coated with 20 $\mu \mathrm{M} A \beta 1-42$ or scrambled control peptide (ScA $\beta 1-42)$. The shear rate utilized was 1,000 $\mathrm{sec}^{-1}$, which leads to thrombus formation. Images were taken at 10 minutes ( $\mathrm{min}$ ) of flow and are representative of 4 independent experiments. Images were quantified by assessing the surface area coverage by platelets with Image J. Data are representative of 4 independent experiments. Statistical analysis was performed by oneway ANOVA with Bonferroni post-hoc test. $* P<0.05 . \mathrm{N}=4$ for (A and $\mathrm{C})$. Aggregation experiments were performed by pretreating platelets with 50 $\mathrm{ng} / \mathrm{mL}$ native $\mathrm{LDL}(\mathrm{nLDL})$ or oxLDL (B) or $20 \mu \mathrm{M} \mathrm{A} \beta 1-42$ or scrambled control peptide (ScAß1-42) (D) for $10 \mathrm{~min}$. Low level aggregation was then stimulated with either $10 \mu \mathrm{g} / \mathrm{mL}$ collagen or 0.03 unit/mL thrombin, as indicated. Aggregation data are representative of 3 independent experiments. 
reducing collagen-dependent aggregation and $\mathrm{O}^{--}$formation, in both human (Figure $3 \mathrm{~A}$ and $\mathrm{B}$ ) and mouse (Figure $5 \mathrm{~A})$ platelets. The secondary role of NOX2 in collagen responses is further demonstrated by thrombus formation experiments under physiological flow, which is marginally but significantly reduced by NOX2 inhibition/silencing in human but not mouse blood (compare Figure 4D and Figure $5 \mathrm{~B})$. This discrepancy between thrombus formation in human and mouse blood could be suggestive of a higher involvement of NOX2 in collagen responses of human platelets. In any case, the dominant role of NOX1 in collagen response is clear in our experiments both in human and mouse platelets. Similarly, NOX2 is essential for the signaling of thrombin without NOX1 involvement in human platelets (Figure 2C and D) and limited yet significant involvement in mouse platelets (Figure 5C). This is in agreement with previous indications from our and other groups, ${ }^{14,40}$ but in essential disagreement with a recent study by Delaney et al. in mouse platelets reaching opposite conclusions (i.e. NOX1 involvement in thrombin signaling and NOX2 involvement in collagen signaling). ${ }^{13}$ Some differences can be expected between signaling pathways and redox dependence of human and mouse platelets, which may explain this discrepancy. Overall, combining human and mouse platelet data, it is safe to state that thrombindependent aggregation depends very heavily on NOX2 activity (although a role of NOX1 in thrombin was detected in mouse platelets, which we did not observe in human platelets), while collagen-induced aggregation was predominantly NOX1-dependent with marginal involvement of NOX2 (as shown in our human platelet data). So, although mouse platelets display some engagement of NOX1 in thrombin responses, species-specific differences cannot fully explain the discrepancy between our report and Delaney et al.'s work. The fact that Delaney et al. used male animals for NOX1 studies and females for NOX2 studies may explain some of the differences with our study (performed entirely on female animals). Other potentially important differences are in the platelet isolation procedure, the concentration of agonists (very low concentration of thrombin used), and the use of collagen-related peptide instead of collagen.

In addition to physiological stimuli, in this study we analyzed the effect of oxLDL and A $\beta 1-42$, platelet modulators associated with the thrombotic complications of atherosclerosis ${ }^{41}$ and cerebrovascular amyloid angiopathy (CAA),42 respectively. Both oxLDL and A $\beta 1-42$ have been shown to activate platelets and act as positive modulators. ${ }^{15,43}$ We confirmed the ability of these molecules to induce partial platelet aggregation. On the other hand, they were able to significantly increase the responses to low levels of physiological agonists. This mode of action is consistent with the definition of positive modulators or primers, ${ }^{44}$ which are characterized by the ability to trigger an unwanted hemostatic response and clot formation leading to thrombosis. The involvement of platelet positive modulators in thrombotic complications associated with diseases is particularly important for vascular health and relative pharmacotherapy. ${ }^{45}$ Interestingly, the aggregation induced by these primers was also redox-dependent and inhibited by the O2*- scavenger PEG-SOD. This is in agreement with previous literature on oxLDL15, ${ }^{46}$ and $A \beta 1-42,{ }^{47}$ and supports the hypothesis that platelet primers may act in a redox-dependent manner. ${ }^{48}$
Also intriguing were our conclusions regarding the involvement of NOX1 and NOX2 in the signaling of oxLDL and A $\beta 1-42$. We provide compelling evidence in human platelets with NOX-selective inhibitors Nox2dstat and NoxA1ds or in genetically modified mouse platelets $\left(\mathrm{NOX}^{-/}\right.$or $\mathrm{NOX} 2^{--}$) that both NOX1 and NOX2 are activated by oxLDL and A $\beta 1-42$ and that they are both required for the functional effects of these pathology-associated modulators on platelets. These conclusions were confirmed by experiments with transgenic mouse platelets, both in thrombus adhesion and aggregation experiments. Only adhesion to A $\beta 1-42$ under low shear seemed exclusively NOX1-dependent. This is a low level adhesion response incapable of properly triggering thrombus formation (Online Supplementary Figure S11). These data may, therefore, suggest a differential involvement of NOX1 and NOX2 in different molecular events triggered by $A \beta 1-42$. This hypothesis merits further study for satisfactory elucidation. The involvement of NOX2 in the signaling of oxLDL has been suggested previously, ${ }^{15,46}$ while the involvement of NOX1 is novel. The possibility of abolishing the effect of oxLDL and A $\beta 1-42$ on platelets by inhibiting only one of the two NOX may suggest that both enzymes are required for reaching a threshold in the superoxide anion levels leading to platelet stimulation. The similarities between the activity and redox-dependence of oxLDL and A $\beta 1-42$ may suggest that they act on similar receptors. As suggested by literature for both molecules, the receptors for these platelet modulators are likely to be CD36. ${ }^{15,49}$ Importantly, the fact that both platelet NOX are required for the modulatory activity of oxLDL or A $\beta 1-42$ offers the opportunity of targeted intervention without the complete inhibition of the response to physiological agonists. In other words, the inhibition of only one NOX isozyme could reduce the pro-thrombotic tendencies associated with vascular inflammation without completely impairing the hemostatic response (i.e. NOX1 inhibition will not impair thrombin response, while NOX2 inhibition will not impair collagen responses). This is the ultimate goal of modern antiplatelet drug development and may help to resolve the persisting problem of bleeding risks associated with all existing antiplatelet treatment. ${ }^{50}$

In summary, herein we describe the development and application of a novel approach to simultaneously monitor the generation of oxygen radicals and platelet aggregation. This technique has the potential to become a standard technique to assess platelet responsiveness and thrombotic risks associated to vascular conditions in a clinical setting. In addition, this study allowed the identification of patterns of platelet regulation and the clarification of the mode of action of physiological agonists and pathological modulators of platelets. The results of this study also highlight the potential of NADPH oxidase targeting for the development of novel antiplatelet drugs with better pharmacodynamic profiles (i.e. limited bleeding side effects).

\footnotetext{
Acknowledgments

The authors would like to thank the British Heart Foundation for sponsoring this research (PG/15/40/31522), the Clinical Research Facility (CRF) of the University of Exeter and in particular Dr Bridget Knight for blood collections and Dr Bruno Fink from Noxygen Science Transfer \& Diagnostics GmbH for the technical support.
} 


\section{References}

1. Chaudhari K, Hamad B, Syed BA. Antithrombotic drugs market. Nat Rev Drug Discov. 2014;13(8):571-572.

2. Stegner D, Nieswandt B. Platelet receptor signaling in thrombus formation. J Mol Med (Berl). 2011;89(2):109-121.

3. Iuliano L, Colavita AR, Leo R, Pratico D, Violi F. Oxygen free radicals and platelet activation. Free Radic Biol Med. 1997;22(6):999-1006.

4. Watt J, Ewart M-A, Greig FH, Oldroyd KG, Wadsworth RM, Kennedy S. The effect of reactive oxygen species on whole blood aggregation and the endothelial cellplatelet interaction in patients with coronary heart disease. Thromb Res. 2012;130(2):210-215

5. Freedman JE. Oxidative stress and platelets. Arterioscler Thromb Vasc Biol. 2008;28(3):s11-16.

6. Ferroni P, Vazzana N, Riondino S, Cuccurullo C, Guadagni F, Davi G. Platelet function in health and disease: from molecular mechanisms, redox considerations to novel therapeutic opportunities. Antioxid Redox Signal. 2012;17(10):1447-1485.

7. Krotz F, Sohn HY, Pohl U. Reactive oxygen species: players in the platelet game. Arterioscler Thromb Vasc Biol. 2004;24(11):1988-1996.

8. Chondrogianni N, Petropoulos I, Grimm S, et al. Protein damage, repair and proteolysis. Mol Aspects Med. 2014;35:1-71.

9. Violi F, Pignatelli P. Platelet NOX, a novel target for anti-thrombotic treatment. Thromb Haemost. 2014;111(5):817-823.

10. Bartimoccia S, Carnevale R, Sanguigni V, et al. NOX 5 is expressed in platelets from patients with chronic granulomatous disease. Thromb Haemost. 2016;116(1):198200.

11. Krotz F, Sohn HY, Gloe T, et al. NAD(P)H oxidase-dependent platelet superoxide anion release increases platelet recruitment. Blood. 2002;100(3):917-924.

12. Begonja AJ, Gambaryan S, Geiger J, et al. Platelet $\mathrm{NAD}(\mathrm{P}) \mathrm{H}$-oxidase-generated ROS production regulates alphallbbeta3-integrin activation independent of the NO/cGMP pathway. Blood. 2005; 106(8):2757-2760.

13. Delaney MK, Kim K, Estevez B, et al. Differential Roles of the NADPH-Oxidase 1 and 2 in Platelet Activation and Thrombosis. Arterioscler Thromb Vasc Biol. 2016;36(5):846-854

14. Vara D, Campanella M, Pula G. The novel NOX inhibitor 2-acetylphenothiazine impairs collagen-dependent thrombus formation in a GPVI-dependent manner. Br J Pharmacol. 2013;168(1):212-224.

15. Magwenzi S, Woodward C, Wraith KS, et al. Oxidized LDL activates blood platelets through CD36/NOX2-mediated inhibition of the cGMP/protein kinase G signaling cascade. Blood. 2015;125(17):2693-2703.

16. Dikalov SI, Harrison DG. Methods for detection of mitochondrial and cellular reactive oxygen species. Antioxid Redox Signal. 2014;20(2):372-382

17. Griendling KK, Touyz RM, Zweier JL, et al. Measurement of Reactive Oxygen Species, Reactive Nitrogen Species, and RedoxDependent Signaling in the Cardiovascular System: A Scientific Statement From the American Heart Association. Circ Res. 2016;119(5):e39-75.

18. Koltai K, Kesmarky G, Feher G, Tibold A,
Toth K. Platelet Aggregometry Testing Molecular Mechanisms, Techniques and Clinical Implications. Int J Mol Sci. 2017;18(8):1803.

19. Davies MJ. Detection and characterisation of radicals using electron paramagnetic resonance (EPR) spin trapping and related methods. Methods. 2016;109:21-30.

20. Hawkins CL, Davies MJ. Detection and characterisation of radicals in biological materials using EPR methodology. Biochim Biophys Acta. 2014;1840(2):708-721

21. Bedard K, Whitehouse S, Jaquet V. Challenges, Progresses, and Promises for Developing Future NADPH Oxidase Therapeutics. Antioxid Redox Signal. 2015:23(5):355-357.

22. Diebold BA, Smith SM, Li Y, Lambeth JD NOX2 As a Target for Drug Development: Indications, Possible Complications, and Progress. Antioxid Redox Signal. 2015;23(5):375-405.

23. Pudusseri A, Shameem R, Spyropoulos AC. A new paradigm shift in antithrombotic therapy. Front Pharmacol. 2013;4:133.

24. Wong PC, Seiffert D, Bird JE, et al. Blockade of protease-activated receptor- 4 (PAR4) provides robust antithrombotic activity with low bleeding. Sci Transl Med. 2017;9(371)

25. Pietraforte D, Vona R, Marchesi A, et al Redox control of platelet functions in physiology and pathophysiology. Antioxid Redox Signal. 2014;21(1):177-193.

26. Ranayhossaini DJ, Rodriguez AI, Sahoo S, et al. Selective recapitulation of conserved and nonconserved regions of putative NOXA1 protein activation domain confers isoform-specific inhibition of Nox1 oxidase and attenuation of endothelial cell migration. J Biol Chem. 2013;288(51):3643736450.

27. Rey FE, Cifuentes ME, Kiarash A, Quinn MT, Pagano PJ. Novel competitive inhibitor of $\mathrm{NAD}(\mathrm{P}) \mathrm{H}$ oxidase assembly attenuates vascular $\mathrm{O}(2)(-)$ and systolic blood pressure in mice. Circ Res. 2001;89(5):408-414.

28. Csanyi G, Cifuentes-Pagano E, Al Ghouleh I, et al. Nox2 B-loop peptide, Nox2ds, specifically inhibits the NADPH oxidase Nox2. Free Radic Biol Med. 2011;51(6):1116-1125.

29. Valente AJ, Jamali AE, Epperson TK Gamez MJ, Pearson DW, Clark RA. NOX1 NADPH oxidase regulation by the NOXA1 SH3 domain. Free Radic Biol Med. 2007;43(3):384-396

30. Lopez JJ, Salido GM, Gomez-Arteta E, Rosado JA, Pariente JA. Thrombin induces apoptotic events through the generation of reactive oxygen species in human platelets. I Thromb Haemost. 2007:5(6):1283-1291.

31. Pignatelli P, Pulcinelli FM, Lenti L, Gazzaniga PP, Violi F. Hydrogen peroxide is involved in collagen-induced platelet activation. Blood. 1998;91(2):484-490.

32. Qiao J, Arthur JF, Gardiner EE, Andrews RK, Zeng L, Xu K. Regulation of platelet activation and thrombus formation by reactive oxygen species. Redox Biol. 2018:14:126-130

33. Chlopicki S, Olszanecki R, Janiszewski M Laurindo FRM, Panz T, Miedzobrodzki J. Functional Role of NADPH Oxidase in Activation of Platelets. Antioxid Redox Signal. 2004;6(4):691-698.

34. Redondo PC, Jardin I, Hernandez-Cruz JM, Pariente JA, Salido GM, Rosado JA. Hydrogen peroxide and peroxynitrite enhance $\mathrm{Ca} 2+$ mobilization and aggregation in platelets from type 2 diabetic patients. Biochem Biophys Res Commun. 2005;333(3):794-802.

35. Furie B, Flaumenhaft R. Thiol isomerases in thrombus formation. Circ Res. 2014; 114(7):1162-1173

36. Jang JY, Min JH, Wang SB, et al. Resveratrol inhibits collagen-induced platelet stimulation through suppressing NADPH oxidase and oxidative inactivation of $\mathrm{SH} 2$ domaincontaining protein tyrosine phosphatase- 2 . Free Radic Biol Med. 2015;89:842-851.

37. Jang JY, Min JH, Chae YH, et al. Reactive oxygen species play a critical role in collagen-induced platelet activation via SHP-2 oxidation. Antioxid Redox Signal. 2014;20(16):2528-2540

38. Mazharian A, Mori J, Wang YJ, et al. Megakaryocyte-specific deletion of the protein-tyrosine phosphatases Shp1 and Shp2 causes abnormal megakaryocyte development, platelet production, and function. Blood. 2013;121(20):4205-4220.

39. Wang SB, Jang JY, Chae YH, et al Kaempferol suppresses collagen-induced platelet activation by inhibiting NADPH oxidase and protecting SHP-2 from oxidative inactivation. Free Radic Biol Med. 2015;83:41-53.

40. Walsh TG, Berndt MC, Carrim N, Cowman J, Kenny D, Metharom P. The role of Nox1 and Nox2 in GPVI-dependent platelet activation and thrombus formation. Redox Biol. 2014;2:178-186.

41. Obermayer G, Afonyushkin T, Binder CJ Oxidized low-density lipoprotein in inflammation-driven thrombosis. Thromb Haemost. 2018;16(3):418-428.

42. Jaunmuktane Z, Mead S, Ellis M, et al. Evidence for human transmission of amyloid-beta pathology and cerebral amyloid angiopathy. Nature. 2015;525(7568):247250

43. Donner L, Falker K, Gremer L, et al. Platelets contribute to amyloid-beta aggregation in cerebral vessels through integrin alphaIIbbeta3-induced outside-in signaling and clusterin release. Sci Signal. 2016; 9(429):ra52

44. Blair TA, Moore SF, Hers I. Circulating primers enhance platelet function and induce resistance to antiplatelet therapy. Thromb Haemost. 2015;13(8):1479-1493.

45. Gresele P, Falcinelli E, Momi S. Potentiation and priming of platelet activation: a potential target for antiplatelet therapy. Trends Pharmacol Sci. 2008:29(7):352-60

46. Carnevale R, Bartimoccia S, Nocella C, et al. LDL oxidation by platelets propagates platelet activation via an oxidative stressmediated mechanism. Atherosclerosis. 2014;237(1):108-116.

47. Abubaker AA, Vara D, Eggleston I Canobbio I, Pula G. A novel flow cytometry assay using dihydroethidium as redoxsensitive probe reveals NADPH oxidasedependent generation of superoxide anion in human platelets exposed to amyloid peptide beta. Platelets. 2017:5:1-9.

48. Dayal S, Wilson KM, Motto DG, Miller FJ Jr., Chauhan AK, Lentz SR. Hydrogen peroxide promotes aging-related platelet hyperactivation and thrombosis Circulation. 2013;127(12):1308-1316.

49. Herczenik E, Bouma B, Korporaal SJ, et al. Activation of human platelets by misfolded proteins. Arterioscler Thromb Vasc Biol. 2007;27(7):1657-1665

50. McFadyen JD, Schaff M, Peter K. Current and future antiplatelet therapies: emphasis on preserving haemostasis. Nat Rev Cardiol. 2018;15(3):181-191. 